\title{
Proopiomelanocortin Neurons in Nucleus Tractus Solitarius Are Activated by Visceral Afferents: Regulation by Cholecystokinin and Opioids
}

\author{
Suzanne M. Appleyard, ${ }^{1,2}$ Timothy W. Bailey, ${ }^{1}$ Mark W. Doyle, ${ }^{1}$ Young-Ho Jin, ${ }^{1}$ James L. Smart, ${ }^{2}$ Malcolm J. Low, $, 2,3,4$ and \\ Michael C. Andresen ${ }^{1}$ \\ ${ }^{1}$ Department of Physiology and Pharmacology, ${ }^{2}$ Vollum Institute, ${ }^{3}$ Department of Behavioral Neuroscience, and ${ }^{4}$ Center for the Study of Weight Regulation \\ and Associated Disorders, Oregon Health and Science University, Portland, Oregon 97239-3098
}

The nucleus tractus solitarius (NTS) receives dense terminations from cranial visceral afferents, including those from the gastrointestinal (GI) system. Although the NTS integrates peripheral satiety signals and relays this signal to central feeding centers, little is known about which NTS neurons are involved or what mechanisms are responsible. Proopiomelanocortin (POMC) neurons are good candidates for GI integration, because disruption of the POMC gene leads to severe obesity and hyperphagia. Here, we used POMC-enhanced green fluorescent protein (EGFP) transgenic mice to identify NTS POMC neurons. Intraperitoneal administration of cholecystokinin (CCK) induced c-fos gene expression in NTS POMC-EGFP neurons, suggesting that they are activated by afferents stimulated by the satiety hormone. We tested the synaptic relationship of these neurons to visceral afferents and their modulation by CCK and opioids using patch recordings in horizontal brain slices. Electrical activation of the solitary tract (ST) evoked EPSCs in NTS POMC-EGFP neurons. The invariant latencies, low failure rates, and substantial paired-pulse depression of the ST-evoked EPSCs indicate that NTS POMC-EGFP neurons are second-order neurons directly contacted by afferent terminals. The EPSCs were blocked by the glutamate antagonist 2,3dihydroxy-6-nitro-7-sulfonyl-benzo[f]quinoxaline. CCK increased the amplitude of the ST-stimulated EPSCs and the frequency of miniature EPSCs, effects attenuated by the CCK1 receptor antagonist lorglumide. In contrast, the orexigenic opioid agonists [D-Ala(2), $\mathrm{N}$-Me-Phe(4), Gly-ol(5)]-enkephalin and met-enkephalin inhibited both ST-stimulated EPSCs and the frequency of miniature EPSCs. These findings identify a potential satiety pathway in which visceral afferents directly activate NTS POMC-EGFP neurons with excitatory inputs that are appropriately modulated by appetite regulators.

Key words: POMC; cholecystokinin; vagus; NTS; food intake; autonomic; enkephalin

\section{Introduction}

Obesity is a major health problem with multiple underlying etiologies. Mutations in the proopiomelanocortin (POMC) and melanocortin-4 (MC4) receptor genes produce severe obesity (Clement et al., 2002). The POMC system comprises two groups of neurons located in the arcuate nucleus of the hypothalamus (ARC) and the nucleus tractus solitarius (NTS) in the medulla. The ARC-POMC system regulates energy homeostasis via widespread limbic, motor, and autonomic projections and is modulated by appetite-regulating hormones such as leptin and glucose (Cone et al., 2001; Cowley et al., 2001; Spiegelman and Flier,

Received 0ct. 7, 2004; revised Feb. 18, 2005; accepted Feb. 24, 2005.

This work was supported by National Institutes of Health Grants DK66604, DK63040, and HL41119. M.J.L. declares a financial interest in transgenic mouse models using the POMC neural regulatory regions, and this potentia conflict of interest has been disclosed to and managed by the Oregon Health and Science University Office for Integrity in Research.

Correspondence should be addressed to Dr. Suzanne M. Appleyard, Vollum Institute, L474 and Department of Physiology and Pharmacology, Oregon Health and Science University, 3183 Southwest Sam Jackson Park Road, Portland, 0R 97239-3098.E-mail: appleyas@ohsu.edu.

M. W. Doyle's present address: Department of Biology, George Fox University, Newberg, OR 97132-2697.

DOI:10.1523/JNEUROSCI.4177-04.2005

Copyright $\odot 2005$ Society for Neuroscience $\quad$ 0270-6474/05/253578-08\$15.00/0
2001; Ibrahim et al., 2003). However, little is known about NTS POMC neurons.

The NTS is the major portal through which visceral afferent information for homeostatic reflexes enters the brain. Vagal afferents from the gastrointestinal (GI) tract synapse within subregions of the NTS, and the activation of these afferents inhibits food intake (Schwartz, 2000; Broberger and Hokfelt, 2001). The NTS, in turn, is reciprocally connected to regions of the brain integral to the regulation of feeding, such as the hypothalamus, amygdala, and nucleus accumbens (Schwartz, 2000; Broberger and Hokfelt, 2001). The medial NTS, in which NTS POMC neurons are concentrated, contains highly fenestrated endothelial cells providing a permeant blood-brain barrier (Gross et al., 1990) and the potential for neurohumoral integration with the systemic circulation. However, despite their strategic location, it is not known whether NTS POMC neurons are regulated by visceral afferents or which satiety factors are involved.

Cholecystokinin (CCK) is released after a meal and inhibits food intake in part by increasing the firing rate of vagal afferents projecting to the NTS (Moran et al., 2001). CCK may interact with the brainstem POMC system, because CCK-induced satiety is blocked by fourth-ventricle injection of a melanocortin antag- 
onist and is absent in MC4 receptor knock-out mice (Fan et al., 2004). However, the site of action of CCK, the role of the vagal afferents, and the cellular mechanisms underlying CCK activation of POMC neurons are not known.

Appetite stimulators are also important in energy homeostasis, and, if NTS POMC neurons are involved, one would predict that they would be targets of orexigenic drugs. Opioids are neuromodulatory peptides that stimulate feeding when injected into the NTS (Kotz et al., 1997). Furthermore, NTS injections of opioid antagonists inhibit food intake, consistent with a basal tone of endogenous opioid action within the nucleus (Kotz et al., 1997, 2000). Because vagal afferent terminals express opioid receptors, afferent regulation of NTS POMC neurons represents an interesting potential target for opioids (Aicher et al., 2000).

We used POMC enhanced green fluorescent protein (EGFP) mice to identify NTS POMC neurons for both in vivo c-fos studies and in a newly developed horizontal brain-slice preparation. Our findings show that POMC-EGFP neurons are directly activated by visceral afferent glutamatergic synapses and that CCK facilitates, and opioids depress, glutamate release onto these neurons. Thus, NTS POMC-EGFP neurons lie in a key position in afferent reflex pathways and respond to signals from two opposing regulators of feeding behavior, supporting the hypothesis that these neurons regulate energy homeostasis.

\section{Materials and Methods}

Breeding, housing, and genotyping of mice. POMC-EGFP transgenic mice were housed on a 14/10 h light/dark cycle at an ambient temperature in the Department of Comparative Medicine murine-specific pathogenfree facility. Mouse chow (Purina Mills, St. Louis, MO) and water were provided ad libitum. Genotyping and breeding of mice were as described previously (Cowley et al., 2001). All animal procedures were conducted with the approval of the Institutional Animal Care and Use Committee in accordance with the United States Public Health Service Policy on Humane Care and Use of Laboratory Animals and the National Institutes of Health Guide for the Care and Use of Laboratory Animals.

CCK induction of $c$-fos. Six- to 8-week-old POMC-EGFP mice were individually housed for $3 \mathrm{~d}$. On the day of the experiment, food was removed $4 \mathrm{~h}$ before the beginning of the dark cycle, and water remained available ad libitum. At the start of the dark cycle, mice received an intraperitoneal injection of either saline or CCK ( 5 or $20 \mu \mathrm{g} / \mathrm{kg}$ doses in saline). Mice were killed $90 \mathrm{~min}$ after the injection, brains were processed, and sections were immunostained for c-fos.

Immunocytochemistry. Anesthetized ( $2 \%$ tribromoethanol) 6- to 8 -week-old mice were perfused transcardially with $4 \%$ paraformaldehyde, and free-floating frozen sections were prepared using a sliding microtome. We processed the sections for immunofluorescence and colocalization of GFP fluorescence using standard techniques (Cowley et al., 2001). Rabbit anti-c-fos (Santa Cruz Biotechnology, Santa Cruz, CA) was used at a final dilution of 1:1000 v/v. After rinsing, sections were incubated in biotinylated horse anti-mouse/rabbit IgG $(10 \mu \mathrm{g} / \mathrm{ml})$ (Vector Laboratories, Burlingame, CA), followed by cyanine 3-conjugated streptavidin (1:500 v/v; Jackson ImmunoResearch, West Grove, PA). Highresolution confocal images were acquired using an Olympus Optical (Tokyo, Japan) FluoView FV300 confocal laser-scanning microscope.

NTS slices. Hindbrains of male POMC-EGFP mice (6-12 weeks old) were prepared as described previously for rats (Doyle and Andresen, 2001; Doyle et al., 2004). Briefly, horizontal slices (250 $\mu \mathrm{m}$ thick) that contained the ST in the same plane as the NTS were cut with a sapphire knife (Delaware Diamond Knives, Wilmington, DE) and mounted in a vibrating microtome (model VT1000 S; Leica Microsystems, Bannockburn, IL). Slices were submerged in a perfusion chamber, and all recordings were performed in artificial CSF composed of the following (in $\mathrm{mM}$ ): $125 \mathrm{NaCl}, 3 \mathrm{KCl}, 1.2 \mathrm{KH}_{2} \mathrm{PO}_{4}, 1.2 \mathrm{MgSO}_{4} 25 \mathrm{NaHCO}_{3}, 10$ dextrose, and $2 \mathrm{CaCl}_{2}, \mathrm{pH} 7.4$, bubbled with $95 \% \mathrm{O}_{2} / 5 \% \mathrm{CO}_{2}$ at $31-35^{\circ} \mathrm{C}$. Recording electrodes were filled with a solution composed of the following (in $\mathrm{mM}$ ):
$10 \mathrm{NaCl}, 130 \mathrm{~K}$-gluconate, 11 EGTA, $1 \mathrm{CaCl}_{2}, 2 \mathrm{MgCl}_{2}, 10 \mathrm{HEPES}, 2$ ATP, and 0.2 GTP, pH 7.3 (295-299 mOsm). Neurons were recorded from NTS within $200 \mu \mathrm{m}$ caudal to obex and medial to the ST. Patch electrodes (3.0-4.5 M $\Omega$ ) were guided to neurons using both fluorescence and differential interference contrast (DIC) optics illuminated with infrared light (Axioskop FS2; Zeiss, Oberkochen, Germany). Voltage-clamp and current-clamp recordings were made with an Axopatch $200 \mathrm{~B}$ or Axoclamp $2 \mathrm{~A}$ amplifier and pClamp software versions 8 and 9 (Axon Instruments, Union City, CA). Only neurons with holding currents not exceeding $100 \mathrm{pA}$ at a $V_{\mathrm{h}}$ of $-60 \mathrm{mV}$ for the $15 \mathrm{~min}$ control period (input resistance, $>150 \mathrm{M} \Omega$ ) were studied further. Synaptic currents were evoked with an ultrafine concentric bipolar stimulating electrode ( $200 \mu \mathrm{m}$; FHC, Bowdoinham, ME) placed on the ST 1-4 mm from the recording electrode. Electrical stimuli were delivered from an isolated programmable stimulator (Master-8; A.M.P.I., Jerusalem, Israel) triggered to deliver a burst of stimuli $(50-200 \mathrm{~Hz})$. Voltage-clamp protocols included $200 \mathrm{~ms}$ voltage-command steps ranging from -80 to $0 \mathrm{mV}$.

2,3-Dihydroxy-6-nitro-7-sulfonyl-benzo[f]quinoxaline (NBQX) and bicuculline methiodide were obtained from Tocris Cookson (Ballwin, MO). CCK, met-enkephalin (ME), naloxone, [D-Ala(2), N-Me-Phe(4), Gly-ol(5)]-enkephalin (DAMGO), and lorglumide were obtained from Sigma (St. Louis, MO), and TTX was obtained from Alomone Labs (Jerusalem, Israel). All drugs were applied for a $5 \mathrm{~min}$ period. In the case of pretreatment with antagonists, the antagonist was applied alone for 5 $\mathrm{min}$, and then the agonist was coapplied for an additional $5 \mathrm{~min}$.

All data are presented as means \pm SEM. Differences in drug effects were tested by repeated-measures ANOVA (RMA) with Bonferroni's post hoc test or Student's $t$ test. Cumulative distributions of miniature synaptic current amplitudes and frequencies were compared using the Kolmogorov-Smirnov (KS) nonparametric analysis. Differences were considered statistically significant for $p$ values $<0.05$.

\section{Results}

\section{Selective distribution of POMC-EGFP neurons within caudal} subnuclei of the NTS

EGFP neurons were widely distributed throughout the caudal NTS from approximately -7.3 to $-7.8 \mathrm{~mm}$ caudal to bregma between the ST and central canal, including the medial, intermediate, commissural, and ventral subdivisions of the NTS (Paxinos, 2001) (Fig. 1 A). EGFP-labeled neurons were most abundant caudal to the area postrema (AP). A small population of the EGFP neurons was also found in the AP itself. This distribution of EGFP neurons is similar to immunohistochemically identified POMC neurons in the rat brainstem (Joseph et al., 1983; Schwartzberg and Nakane, 1983; Bronstein et al., 1992). The bright fluorescence made POMC-EGFP neurons easily identifiable for recording (Fig. 2C-E).

\section{CCK activates c-fos expression in NTS POMC-EGFP neurons}

To test whether CCK activates NTS POMC-EGFP neurons in vivo, we examined the induction of c-fos expression. Intraperitoneal injections of CCK or saline were administered to mice at lights off, and the tissue was collected 90 min later. Food was withheld $4 \mathrm{~h}$ before and after the injections to avoid the confounding effect of c-fos activation secondary to feeding itself. c-fos gene expression was remarkably low in POMC-EGFP neurons under control conditions $(<1 \%)$. CCK increased c-fos expression in POMC-EGFP neurons $>50$-fold (Fig. $1 B-E$ ). No additional increase in the number of EGFP neurons activated by CCK was seen at higher doses of CCK (data not shown). CCK also induced c-fos expression in non-POMC-EGFP neurons ( $8 \%$ of total neurons activated by CCK were POMC-EGFP neurons). Thus, systemic CCK, at concentrations known to induce satiety, dosedependently activated NTS POMC-EGFP neurons. 

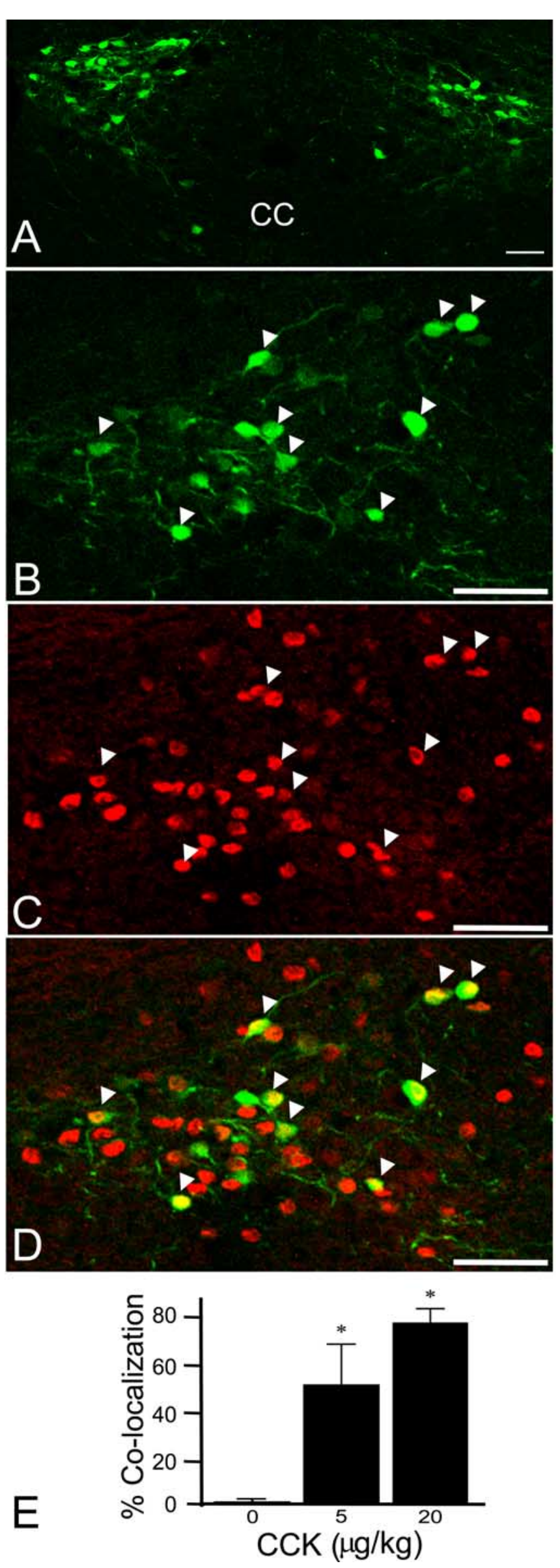

Solitary tract activation evokes monosynaptic responses in NTS POMC-EGFP neurons

NTS POMC-EGFP neurons are most densely located in regions of the caudal NTS that are known to receive vagal afferent synapses. This distribution raises the interesting prospect that these neurons are directly regulated by sensory afferent signals. To test this, we cut brainstem slices horizontally to preserve a lengthy segment of the ST in the same plane as the cell bodies of the NTS (Doyle et al., 2004). The placement of the stimulating electrode on the visible ST distant from the recording area eliminated focal activation of local interneurons and interconnecting fibers (Fig. $2 A, B$ ). Brief shocks (duration, $100 \mu \mathrm{s}$ ) to the ST evoked EPSCs in NTS POMC-EGFP neurons. Such ST-EPSCs were generally unitary all-or-none responses with sharp stimulus-current thresholds and little suprathreshold recruitment above threshold stimulus levels (Fig. $2 \mathrm{~F}$ ). The response characteristics of the STEPSCs in 58 of $72(81 \%)$ NTS POMC-EGFP neurons were consistent with monosynaptic transmission using electrophysiological criteria (Doyle and Andresen, 2001). We combined three measures of synaptic reliability to judge monosynaptic behavior: (1) short absolute latency (1-10 ms), (2) minimal synaptic jitter, the SD of the intraneuronal latency $(<200 \mu \mathrm{s})$, and (3) the absence of synaptic failures at high stimulus frequencies $(50-100$ $\mathrm{Hz}$ ) to a burst of five shocks. Successive shocks (train of five pulses at $50 \mathrm{~Hz}$ ) evoked a frequency-dependent depression of EPSC amplitude.

The non-NMDA glutamate receptor antagonist NBQX blocked all ST-evoked EPSCs (Fig. $2 G)(n=8)$. Fourteen (19\%) NTS POMC-EGFP neurons had a higher synaptic jitter that may reflect polysynaptic events.

\section{CCK facilitates ST afferent transmission}

A proportion of vagal afferents from the GI are known to express CCK1 receptors on their peripheral terminals (Moran et al., 1990; Corp et al., 1993; Moriarty et al., 1997; Broberger et al., 2001), and CCK increases the firing rate of these fibers (Schwartz, 2000; Broberger and Hokfelt, 2001). Interestingly, some vagal afferent terminals in the NTS also express CCK1 receptors (Moran et al., 1990; Corp et al., 1993; Moriarty et al., 1997; Broberger et al., 2001). We therefore tested whether CCK-sensitive afferent terminals activated POMC neurons by determining the effects of CCK on ST inputs onto NTS POMC-EGFP neurons. CCK (100 nM) significantly increased the amplitude of the ST-stimulated EPSC in four of eight (50\%) neurons tested (Fig. 3). In contrast, CCK coapplied with $10 \mu \mathrm{M}$ lorglumide did not change the amplitude of the ST-stimulated EPSC in seven of seven (100\%) neurons tested. Together, these observations suggest that a major portion of the cranial visceral afferents that excite POMC-EGFP neurons are CCK sensitive and that CCK rapidly enhances this excitatory drive. CCK did not significantly change the input resistance of the CCK-sensitive neurons, suggesting a presynaptic

\section{$\leftarrow$}

Figure 1. CCK induces $c$-fos expression in NTS POMC-EGFP neurons. A, Distribution of NTS POMC-EGFP neurons in a coronal section of the mouse brainstem. EGFP-positive neurons appear green. CC, Central canal. $\boldsymbol{B}-\boldsymbol{D}$, C-fos induction by $20 \mu \mathrm{g} / \mathrm{kg}$ CCK. CCK strongly increased c-fos gene expression. $\boldsymbol{B}$, EGFP. $\boldsymbol{C}, \mathrm{c}-$-fos. $\boldsymbol{D}$, Merged image. EGFP-positive neurons appear green, $c$-fos expression appears red, and colocalization appears yellow. Arrowheads indicate examples of colocalization. $E$, Bar graph showing the average number of c-fos-positive POMC-EGFP neurons after different doses of $C\left(K{ }^{*} p<0.05\right.$; Student's $t$ test; compared with saline control). Saline, 2 of 231 neurons ( 9 slices from 4 animals); $5 \mu \mathrm{g} / \mathrm{kg}$ CCK, 64 of 115 neurons ( 6 slices from 3 animals); and $20 \mu \mathrm{g} / \mathrm{kg}$ (CK, 129 of 175 (8 slices from 3 animals). Scale bars: $\boldsymbol{A}, 50 \mu \mathrm{m} ; \boldsymbol{B}-\boldsymbol{D}$, $25 \mu \mathrm{M}$. Error bars represent SEM. 

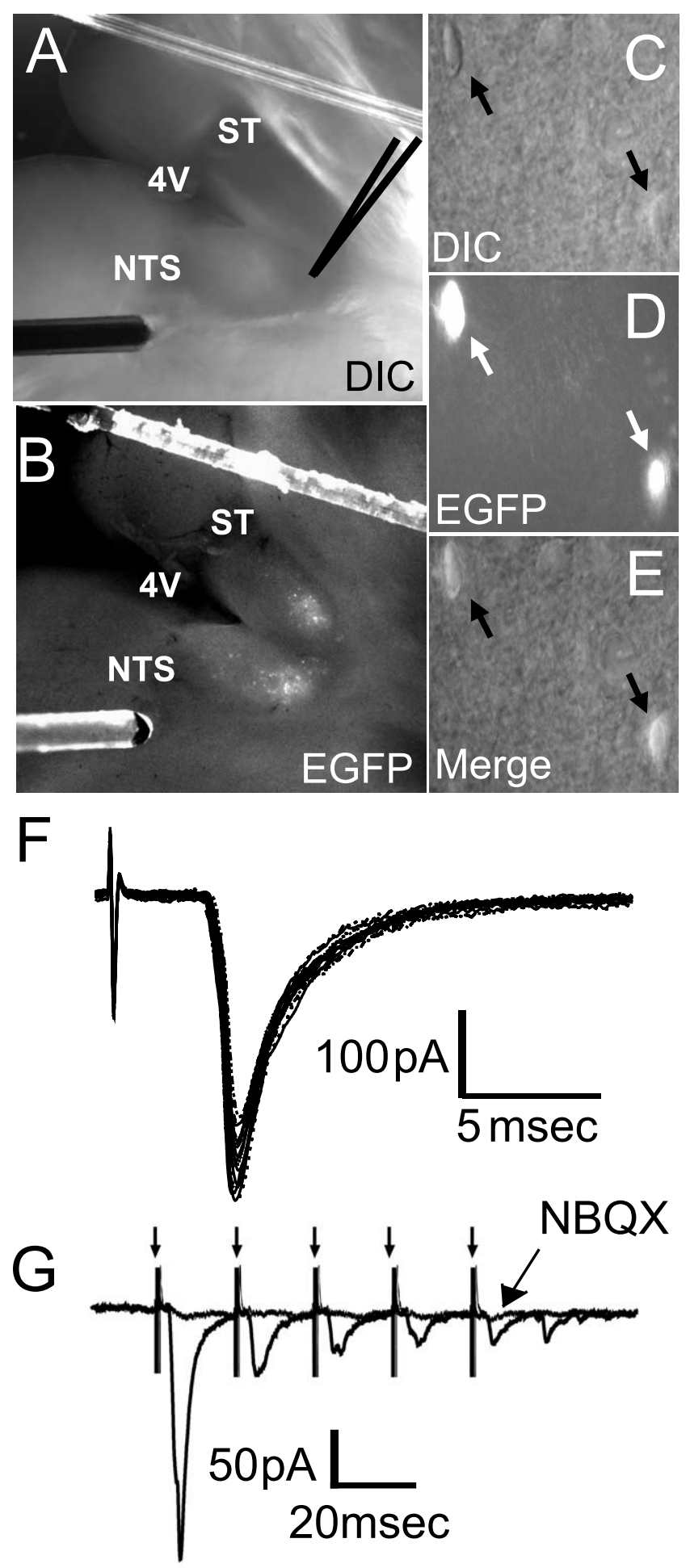

Figure 2. Solitary tract-evoked NTS synaptic responses in NTS POMC-EGFP neurons. $\boldsymbol{A}, \boldsymbol{B}$, Visualization of the NTS brain-slice preparation using both DIC $(\boldsymbol{A})$ and fluorescence $(\boldsymbol{B})$. Orientation of the mouse brainstem slices in the horizontal plane allowed the placement of the concentric bipolar stimulating electrode on the ST several millimeters from the recording region (dark gray center) in the medial NTS. 4V, Fourth ventricle. $\boldsymbol{C}, \boldsymbol{E}$, Identification of individual POMC-EGFP neurons by DIC $(\boldsymbol{C})$, fluorescence $(\boldsymbol{D})$, and merge $(\boldsymbol{E})$. Two POMC-EGFP-positive neurons can be seen (arrows). $\boldsymbol{F}$, ST activation evoked monosynaptic EPSCs in an NTS POMCEGFP neuron; a total of 10 successive EPSCs are shown. $V_{\mathrm{m}}=-60 \mathrm{mV}$. ST shock evoked a short-latency EPSC with high reliability (latency, $1.58 \mathrm{~ms}$; jitter, $65 \mu \mathrm{s}$ and no observed failures at $50 \mathrm{~Hz}$ ST stimulation). G, Successive shocks (train of 5 pulses at $50 \mathrm{~Hz}$; arrows) evoked a frequency-dependent depression of EPSC amplitude. The non-NMDA glutamate receptor antagonist NBQX $(10 \mu \mathrm{M})$ completely blocked the EPSCS.

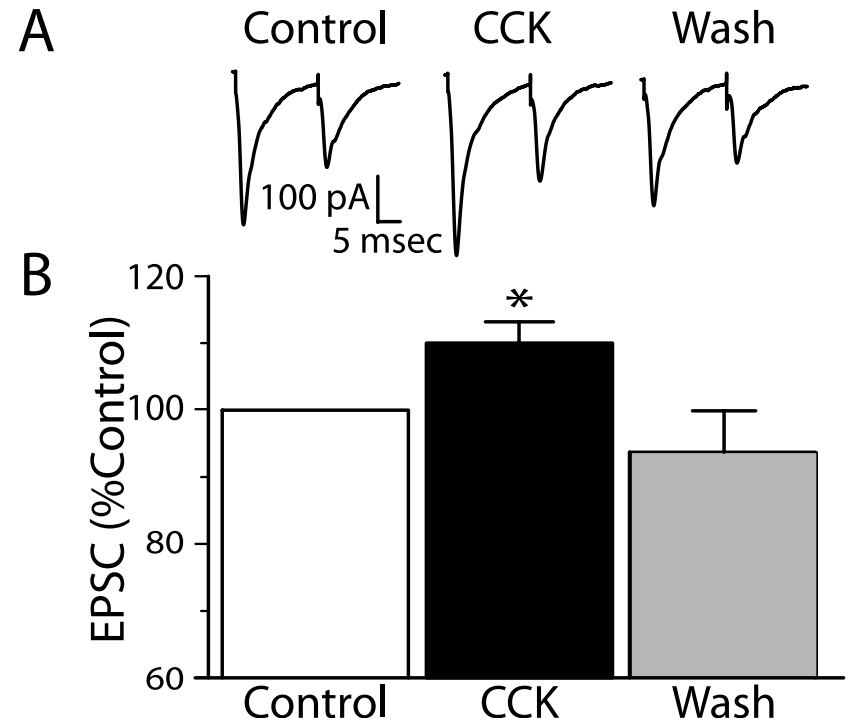

Figure 3. CCK facilitates ST-afferent transmission onto NTS POMC-EGFP neurons. $A$, Representative current traces for control, CCK (100 nM), and after washing (Wash). CCK significantly increased the EPSC amplitude in four of eight neurons tested ( $p<0.05$; Student's $t$ test). $\boldsymbol{B}$, Graph showing the average maximal increase in the ST-stimulated EPSC by CCK $\left[{ }^{*} p<0.05\right.$ (Student's $t$ test) compared with control and Wash]. Error bars represent SEM.

site of action that would be consistent with the distribution of CCK1 receptors to afferent terminals within the NTS (Moran et al., 1990; Corp et al., 1993; Moriarty et al., 1997; Broberger et al., 2001).

Presynaptic CCK1 receptor activation increases miniature EPSCs on NTS POMC-EGFP neurons

To directly determine whether CCK had a presynaptic or postsynaptic mechanism of action, we examined miniature EPSCs (mEPSCs) by blocking evoked synaptic transmission with TTX and blocking GABA-mediated IPSCs with bicuculline. CCK significantly increased the frequency of mEPSCs in all experiments ( $p<0.001 ; \mathrm{KS} ; n=14)$, suggesting a presynaptic mechanism of action. CCK (10 nM) increased mEPSCs an average of 2.6-fold from $0.35 \pm 0.06$ to $0.89 \pm 0.11 \mathrm{~Hz}(n=7)$, an effect reversed by washing to $0.35 \pm 0.08 \mathrm{~Hz}$ (Fig. 4). After $10 \mathrm{~min}$, a second application of $10 \mathrm{~nm}$ CCK again significantly increased the frequency of mEPSCs to the same level as the first response (from $0.35 \pm 0.08$ to $0.95 \pm 0.06 \mathrm{~Hz} ; n=3$ ). In contrast, if the CCK1 receptor antagonist lorglumide $(1 \mu \mathrm{M})$ was coapplied with $10 \mathrm{nM}$ CCK, there was no significant change in mEPSC frequency (from $0.26 \pm 0.02$ to $0.29 \pm 0.07 \mathrm{~Hz} ; n=4)$. CCK $(10 \mathrm{nM}$ ) caused no reversible change in amplitude in any individual experiment (KS test; average amplitudes: control, $24.8 \pm 3.2 \mathrm{pA}$; CCK, $27.0 \pm$ $3.2 \mathrm{pA}$; CCK plus lorglumide, $23.3 \pm 2.7 \mathrm{pA}$ ), indicating no postsynaptic effects of CCK to alter glutamate EPSCs. CCK (100 nM) increased the mean frequency almost fivefold, from $0.25 \pm$ 0.19 to $1.2 \pm 0.3 \mathrm{~Hz}(n=5)$, an effect reversed by coapplication of $10 \mu \mathrm{M}$ lorglumide $(p<0.001 ; \mathrm{KS})$, with the mean frequency returning to $0.37 \pm 0.13(n=3)$. NBQX abolished all mEPSCs, confirming that they were mediated by glutamate. These data, together with the data on ST-stimulated EPSC amplitude, suggest that the increase in glutamate release is at least in part from the solitary tract afferent terminals.

\section{CCK increases the firing rate of NTS POMC-EGFP neurons}

In current clamp, POMC-EGFP neurons had an average resting membrane potential of $71 \pm 1 \mathrm{mV}(n=50)$ and rarely spiked 

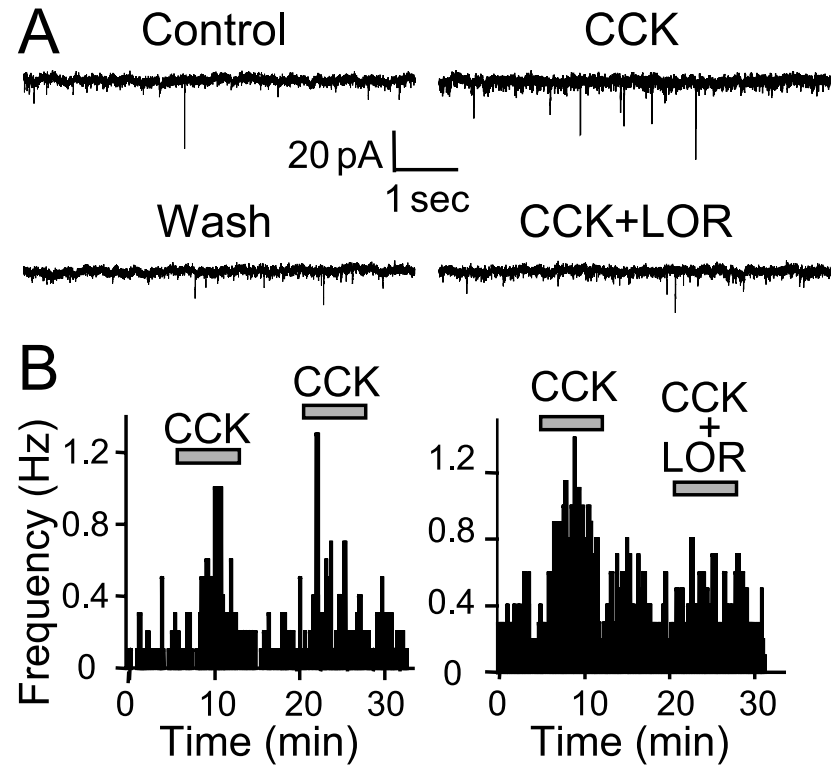

Figure 4. CCK increases the frequency of miniature EPSCS in NTS POMC-EGFP neurons. TTX and bicuculline were included in the external solution for all experiments. $\boldsymbol{A}$, Expanded representative current traces of spontaneous mEPSCs in control, with CCK, after washing (Wash), and with CCK plus lorglumide (LOR). B, Graph showing the frequency of mEPSCs over time. Each bar represents the number of events in a 10 s time period. CCK (10 nM) increased the rate of EPSCS, and this effect was reversed by washing for $10 \mathrm{~min}$. A second application of CCK stimulated the same size increase in mEPSC frequency. In contrast, no increase in mEPSC frequency was seen when the CCK1 receptor antagonist lorglumide $(1 \mu \mathrm{M})$ was coapplied with CCK.

spontaneously (resting firing rate, $0.06 \pm 0.03 \mathrm{~Hz}$ ) (Fig. 5A). CCK (100 nM) rapidly depolarized these neurons on average by $4.2 \pm 0.6 \mathrm{mV}$ and increased their firing rate $>10$-fold to $0.86 \pm$ $0.34 \mathrm{~Hz}(p<0.05 ; \mathrm{RMA} ; n=7)$, an effect reversed after washing or coapplication of $10 \mu \mathrm{M}$ lorglumide $(0.1 \pm 0.34 \mathrm{~Hz} ; p<0.05$; RMA; $n=4)$ (Fig. $5 A)$. CCK (100 pM) consistently depolarized NTS POMC-EGFP neurons, although this much-lower concentration of CCK was rarely sufficient alone to increase firing rates (one of five neurons; data not shown). Under basal conditions, ST stimuli elicited $2 \pm 0.4$ action potentials per train of five stimuli. CCK (100 nM) significantly increased ST-evoked action potentials to an average of $4 \pm 0.2$ per five stimuli ( $p<0.05$; RMA; $n=7$ ) (Fig. 5B). Washing (5-10 min) or coapplication of $10 \mu$ M lorglumide $(2.3 \pm 0.6$ per five stimuli; $p<0.05$; RMA; $n=$ 4) reversed such responses.

Presynaptic $\mu$ receptors inhibit glutamate release

If POMC-EGFP neurons are involved in appetite regulation, orexigenic factors such as opioids might be expected to affect their activation by afferent inputs. ME $(10 \mu \mathrm{M})$ inhibited the ST-EPSC amplitude an average of $55 \pm 6.3 \%(n=5)$, an effect that was reversed after washing (Fig. $6 A, B$ ). Application of the $\mu$-opioid receptor-specific agonist DAMGO (300 nM and $1 \mu \mathrm{M})$ substantially inhibited the ST-EPSC amplitude by $45 \pm 6 \%$, an action blocked by the nonselective opioid antagonist naloxone $(n=7)$ (Fig. $6 C, D)$. In contrast, neither the $\kappa$ receptor-specific agonist $(5 \alpha 7 \alpha, 8 \beta)-(-)-N$ methyl- $N$-(7-(1-pyrrolidinyl)-1-oxaspiro(4,5)dec-8-yl)benzeneacetamide $(\mathrm{U} 69,593)(1 \mu \mathrm{M})$ or the $\delta$-specific agonist $\left[\mathrm{D}-\mathrm{Pen}^{2,5}\right]$ enkephalin $(1 \mu \mathrm{M})$ consistently altered the ST-EPSC amplitude (average inhibition of $10.1 \pm 9.7 \%, n=4$, and $20 \pm 15 \%, n=4$, respectively). DAMGO $(1 \mu \mathrm{M})$ also inhibited the ST-EPSC amplitude in NTS neurons not expressing EGFP $(55 \pm 19 \% ; n=5)$, and this effect was attenuated by $1 \mu \mathrm{m}$ naloxone.
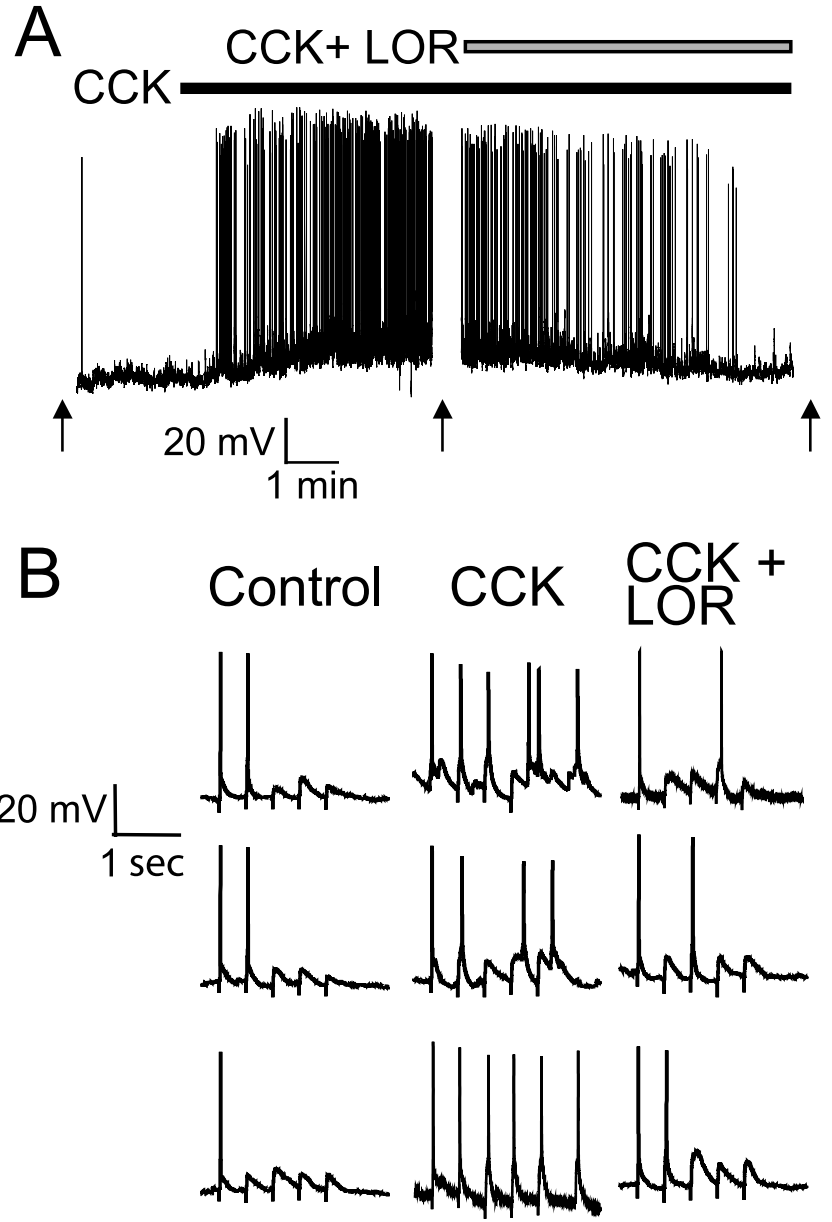

Figure 5. CCK depolarizes NTS POMC-EGFP neurons and increases ST-stimulated action potentials. $\boldsymbol{A}, \boldsymbol{B}$, Voltage traces from an NTS POMC-EGFP neuron. $\boldsymbol{A}$, CCK (100 nM) depolarized POMC neurons by $4.4 \pm 0.6 \mathrm{mV}$ and increased their firing rate within $2-5 \mathrm{~min}(n=7)$. Arrows indicate where ST was stimulated. $\boldsymbol{B}$, CCK (100 nM) increases the number of evoked action potentials after a train of ST stimulation ( $5 \mathrm{at} 5 \mathrm{~Hz}$ ). The effects of $\mathrm{CCK}$ were abolished after either being washed for $10 \mathrm{~min}$ (data not shown) or coapplication of the CCK1 receptor antagonist lorglumide (LOR) $(10 \mu \mathrm{M})$. Three representative traces of each condition are shown.

As an initial index of whether opioids acted presynaptically on the terminals, we examined the paired-pulse ratio. Any change in the ratio of the amplitude of the first stimulated EPSC (P1) and the second stimulated EPSC (P2) generally indicates an effect on the presynaptic terminal. $\mathrm{ME}$ increased the paired-pulse ratio (P2/P1) from $0.6 \pm 0.05$ to $0.81 \pm 0.08(n=5 ; p<0.05$; control vs ME; Student's $t$ test) (Fig. 7A). This effect was reversed after washing to $0.53 \pm 0.04$ (Fig. $6 A)(n=5 ; p<0.01$; ME vs washing; Student's $t$ test).

To better delineate presynaptic opioid mechanisms, we isolated mEPSCs using TTX and bicuculline. Met-enkephalin reversibly decreased mEPSC frequency (ME, $44 \pm 6 \%$ of control frequency; $n=5$ ) consistent with a presynaptic action (Fig. $7 B$ ). This effect was reversed by washing for $5 \mathrm{~min}$ ( $90 \pm 7 \%$ of control; $n=5)$. The $\mu$ receptor-selective agonist DAMGO $(1 \mu \mathrm{M})$ also significantly decreased the frequency of mEPSCs ( $45 \pm 7 \%$ of control frequency; $n=4$ ) (Fig. 6C), an effect attenuated by naloxone (91 $\pm 7 \%$ of control; $n=4$ ) (Fig. $7 C$ ). In all experiments, the decrease in frequency was significant and reversed after either washing or coapplication of an antagonist $(p<0.01$; $\mathrm{KS} ; n=9)$. 


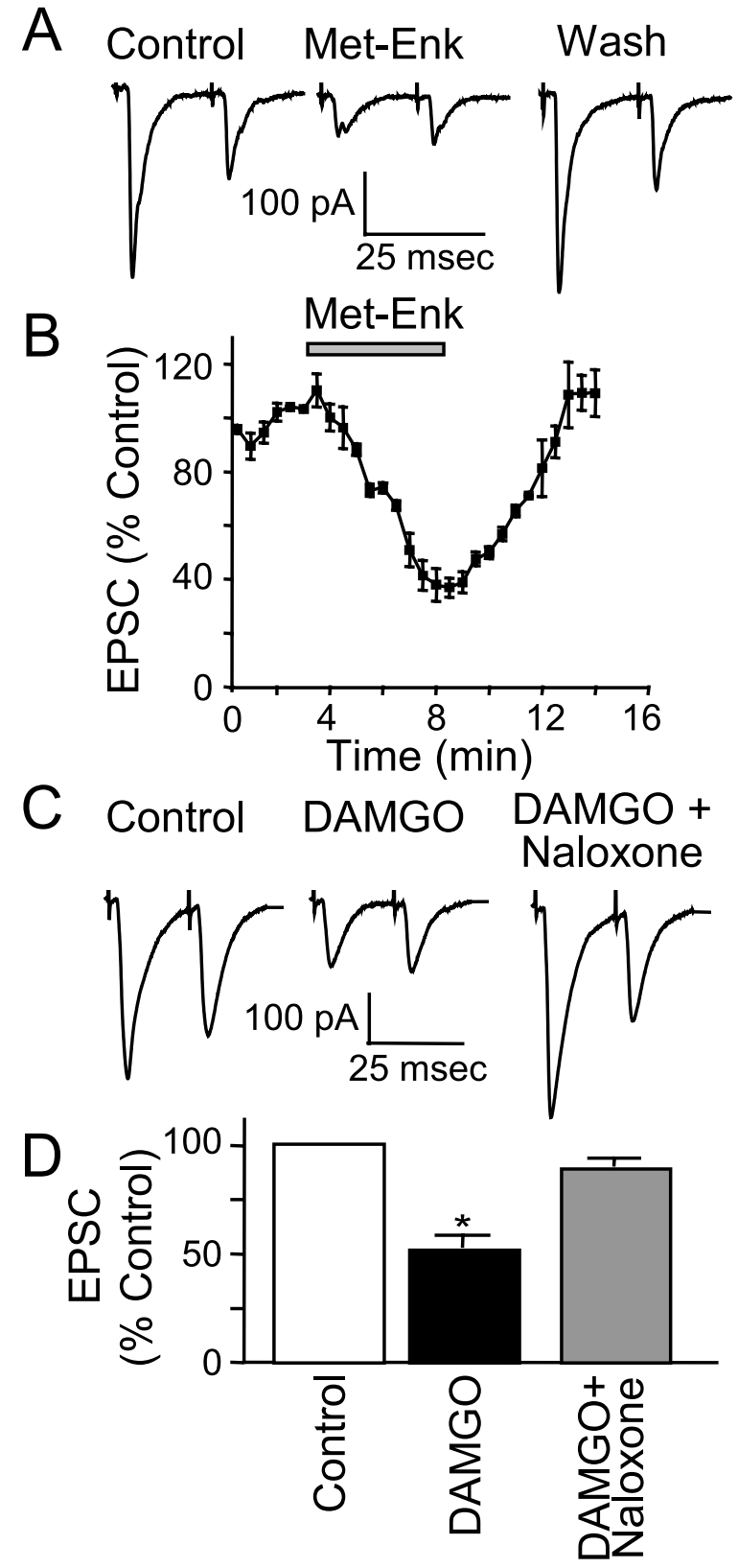

Figure 6. Opioids inhibit ST-stimulated EPSCS. A, Representative current trace for control, met-enkephalin (Met-Enk; $10 \mu \mathrm{M}$ ), and after washing (Wash). Met-Enk inhibited the EPSC amplitude by $55 \pm 6.3 \%$ ( $p<0.05$ compared with control and Wash). B, Graph showing the time course of Met-Enkinhibition of ST-stimulated EPSCs over time. C, Representative current traces showing that DAMGO $(1 \mu \mathrm{M})$ inhibited the control EPSC and that the opioid antagonist naloxone $(1 \mu \mathrm{M})$ reversed this effect. $\boldsymbol{D}$, Graph showing the average maximal inhibition of the ST-stimulated EPSC by DAMGO [ ${ }^{*} p<0.05$ (RMA) compared with control and DAMGO plus naloxone].

\section{Discussion}

The central melanocortin system is well recognized for its importance to energy homeostasis, yet little is known about its brainstem component, the NTS POMC neurons. Three new findings of this study provide key information about how NTS POMCEGFP neurons are regulated: first, they receive direct glutamatergic synapses from visceral afferents, including CCK-sensitive afferents. Second, CCK increased the activation of these neurons in brain slices as well as in vivo. Third, opioids inhibited the afferent activation of these neurons. These findings suggest a potential satiety pathway in which visceral afferents directly activate NTS

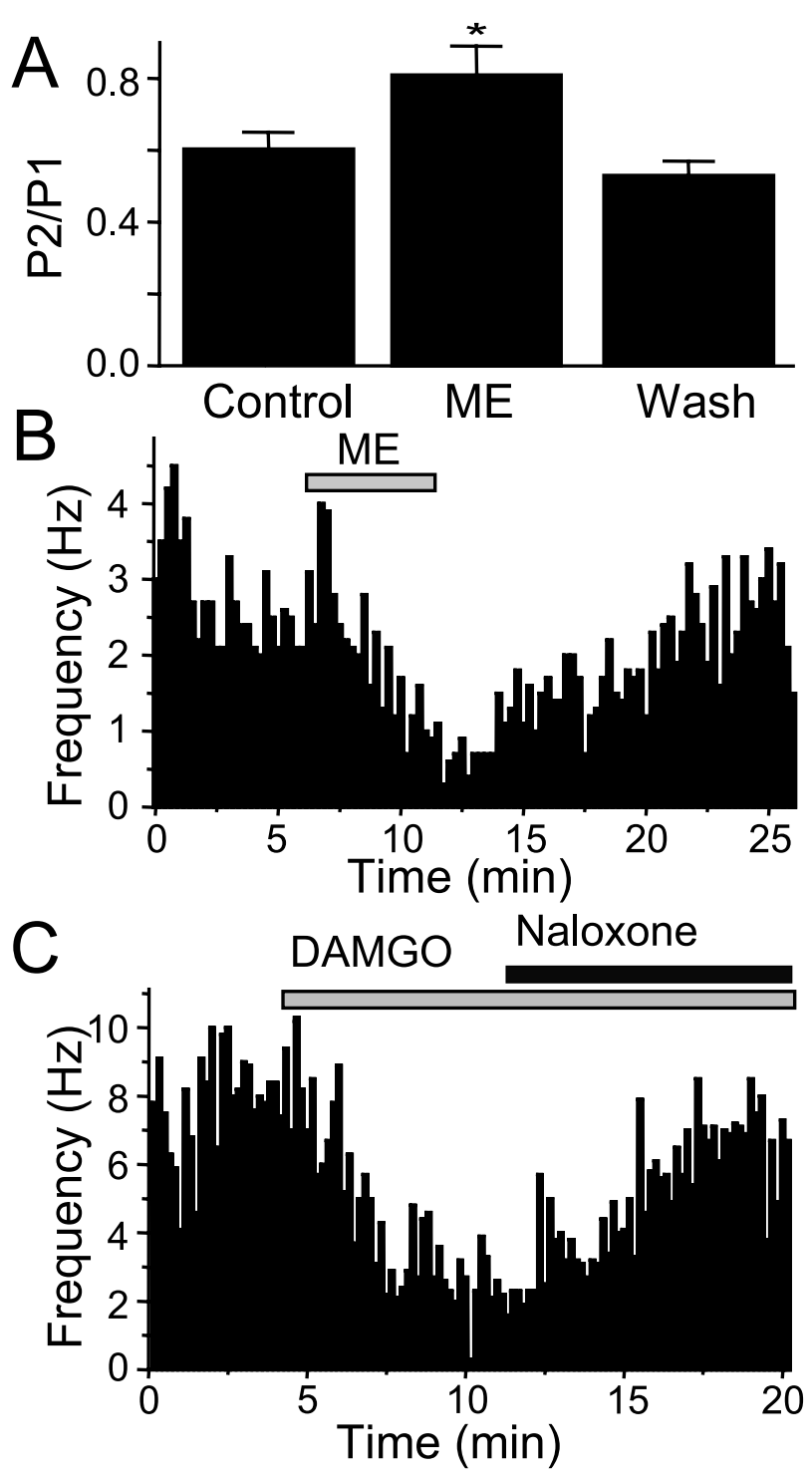

Figure 7. Opioids increase the paired-pulse ratio of evoked EPSCS and decrease the frequency of miniature EPSC $\operatorname{in}$ NTS POMC-EGFP neurons. $A$, Graph showing the paired-pulse ratio of the ST-stimulated EPSC ( $50 \mathrm{~Hz})$ in control, with $10 \mu \mathrm{M} \mathrm{ME}$, and after washing (Wash) [ ${ }^{*} p<0.05$ (Student's $t$ test) compared with control and Wash]. $B$, Graph showing the frequency of $m E P S C$ s over time. ME $(10 \mu \mathrm{M})$ decreased the rate of $\mathrm{mEPSC}$, and this effect was reversed by washing for $10 \mathrm{~min}$. $\boldsymbol{C}$, Graph showing the frequency of mEPSC over time. The $\mu$-opioid agonist DAMGO $(1 \mu \mathrm{M})$ decreased the rate of $\mathrm{mEPSCS}$, and this effect was reversed by coapplication of the opioid antagonist naloxone. NBQX blocked all of the mEPSCs (data not shown).

POMC-EGFP neurons at a primary synapse capable of being appropriately modulated by appetite regulators.

\section{CCK activates NTS POMC-EGFP neurons in vivo}

Systemic administration of CCK to mice activated c-fos gene transcription in more than one-half of POMC-EGFP neurons. This study confirms that POMC-EGFP neurons are strongly activated during a satiety response in vivo and provides a direct link of these specific neurons to systemic eating behavior. Furthermore, the POMC system is required for this behavior, because the satiety effects of CCK were attenuated by MC4 receptor antagonists and absent in MC4 receptor knock-out mice (Fan et al., 2004). However, such observations do not identify the mechanisms or pathways responsible for the stimulation of NTS POMC neurons. 
Visceral afferents activate NTS POMC-EGFP neurons

There has been speculation about whether NTS POMC neurons are activated by incoming afferents; however, this has not been determined previously. Our electrophysiological results show that NTS POMC-EGFP neurons are activated by afferents, with the majority ( $81 \%$ ) being second-order neurons receiving direct afferent synaptic inputs. These data place NTS POMC-EGFP neurons in a key position in the afferent pathways, because any modulation of this first synapse in the CNS provides a mechanism to affect all downstream responses.

\section{CCK-sensitive afferents activate NTS POMC-EGFP neurons in brain slices}

CCK increased the amplitude of ST-stimulated EPSCs in 50\% of NTS POMC-EGFP neurons. The finding that POMC-EGFP neurons are activated by CCK-sensitive afferents suggests that these neurons are directly contacted by GI afferents providing inhibitory feedback on food intake (Schwartz and Moran, 1996). Interestingly, $50 \%$ is the same proportion of POMC-EGFP neurons in which $5 \mu \mathrm{g} / \mathrm{kg}$ CCK induced c-fos activation, suggesting that our in vitro results closely reflect an in vivo pathway. CCK increased the frequency of mEPSC events, suggesting a presynaptic mechanism of action to increase glutamate release onto NTS POMC-EGFP neurons. This result is consistent with CCK binding sites located on the terminals of vagal afferents in the medial NTS (Moran et al., 1990; Corp et al., 1993; Moriarty et al., 1997; Broberger et al., 2001) and the finding that the cell bodies of the afferents terminating in the NTS (located in the nodose ganglia) are activated by CCK (Lankisch et al., 2002). Interestingly, although CCK increased ST-stimulated EPSC amplitude in 50\% of POMC-EGFP neurons, it increased the mEPSC frequency in all neurons examined. The reason for this difference is not clear; however, it suggests the possibility that CCK has effects on both visceral afferent and nonafferent glutamate terminals in the NTS.

Extensive evidence demonstrates that endogenous and exogenous CCK reduce food intake by activating vagal afferents in the periphery (Moran et al., 2001). However, CCK1 receptor antagonists that cross the blood-brain barrier stimulate food intake in vagotomized animals (Reidelberger et al., 2004). Such observations, along with considerable evidence of CNS CCK1 receptor expression, raise the possibility that CCK has both central and peripheral actions (Ritter, 2004). Injections of CCK into the NTS inhibit food intake, showing that central actions can cause physiological effects (Talman et al., 1991; Blevins et al., 2000). CCK has also been shown to both directly and indirectly activate gastric projection neurons in the dorsal motor nucleus of the vagus (Zheng et al., 2005). Although fenestrated capillaries within the NTS may permit circulating CCK to directly modulate NTS POMC-EGFP neurons, it is unlikely that the levels of circulating endogenous CCK would reach the maximal concentrations used in this study. However, local sources of CCK within the NTS raise the possibility of regional release modulating POMC neuronal activity (e.g., fibers within the NTS may release peptide from either local CCK neurons or projections terminating in the NTS) (Takagi et al., 1984). Interestingly, CCK mRNA expression is induced in nodose ganglion neurons after vagotomy (Broberger et al., 2001) so that conceivably, CCK may also be released from sensory afferents under certain conditions.

Our results, together with previous findings, indicate two mechanisms by which CCK may activate the brainstem POMC system. First, CCK may activate the brainstem POMC system indirectly via activation of afferents that we show directly activate NTS POMC-EGFP neurons. Second, CCK may activate the brainstem POMC system via central actions to facilitate glutamate release onto NTS POMC-EGFP neurons.

\section{Opioid agonists inhibit afferent activation of NTS POMC-EGFP neurons}

Opioids have opposing actions to CCK on food intake mediated by actions on multiple brain regions (Levine and Billington, 2004). NTS injections of opioid agonists stimulate food intake, suggesting that opioids regulate satiety in part via actions at the level of the NTS (Kotz et al., 1997, 2000). Here, we show that opioids inhibit afferent activation of NTS POMC-EGFP neurons by presynaptically decreasing glutamate release. Thus, a key orexigenic peptide inhibits excitatory inputs onto NTS POMCEGFP neurons. These effects are mediated via activation of the $\mu$-opioid receptor, consistent with $\mu$ agonists stimulating food intake when microinjected into the NTS (Kotz et al., 1997). Opioid inhibition of afferent inputs was not limited to POMC-EGFP neurons, because unlabeled NTS neurons responded similarly. This NTS mechanism may underlie the behavioral effects of the opioid antagonist naloxone to shorten meal size without changing meal number (Kirkham and Blundell, 1984; Levine and Billington, 2004). Endogenous opioids are likely to modulate this synapse, given that opioid antagonists alter food intake (Kotz et al., 1997, 2000). Endogenous opioids may originate from $\beta$-endorphin being released from the NTS POMC neurons themselves or from enkephalin-positive neurons also located in the NTS (Velley et al., 1991). Whatever the source of the endogenous opioid, regulation of their release would provide a potent mechanism to modulate afferent input and NTS neuronal activity.

\section{Role of NTS POMC-EGFP neurons in the regulation of energy homeostasis}

Substantial evidence indicates that global disruption of the POMC system leads to obesity (Coll et al., 2004). However, these studies cannot distinguish between the ARC and NTS POMC neurons, because both were affected. Our present results provide a potential framework for how NTS POMC-EGFP neurons contribute to a satiety reflex. Visceral afferent activation and its modulation by appetite regulators suggest that NTS POMC neurons play a role in energy homeostasis in addition to the ARC-POMC neurons. Many important details remain to be determined, in particular how NTS POMC neurons interact with the rest of the system. Previous studies described projections from NTS POMC neurons to other medullary nuclei (Palkovits and Eskay, 1987; Palkovits et al., 1987), and it is possible that NTS POMC-EGFP neurons modulate feeding and GI and autonomic activity via such direct projections. Alternatively, local contacts by NTS POMC-EGFP neurons may modulate the activity of other NTS neurons that in turn project to feeding centers. Actions in the brainstem are also important for the effects of many other regulators of energy homeostasis, and future studies will need to address whether these factors also regulate NTS POMC neurons (Grill and Kaplan, 2002; Grill et al., 2002, 2004; Faulconbridge et al., 2003).

\section{Role of NTS POMC-EGFP neurons in the regulation of other autonomic functions}

The ST contains a broad array of sensory afferents, in addition to GI vagal afferents, including cardiac and baroreceptor afferents. NTS POMC-EGFP neurons may receive information from these additional sensory afferents and participate in neuronal circuits widely influencing autonomic functions. Interestingly, melanocortins, opioids, and CCK all modulate heart rate and blood pres- 
sure (Versteeg et al., 1998; Bodnar and Hadjimarkou, 2002; Verberne et al., 2003). Given the epidemiological relationship between obesity and hypertension, NTS POMC-EGFP neurons could provide a bridge between cardiovascular and energy homeostatic regulatory pathways.

In summary, NTS POMC-EGFP neurons are directly activated by CCK-sensitive afferent inputs, and this pathway is regulated by both suppressors and activators of appetite. Thus, the distribution of POMC neurons in both the hypothalamus, a region integral to the long-term regulation of food intake, and the NTS, in which neurons respond to incoming afferents carrying short-term information on satiety, endows the POMC system with the potential to integrate two different feedback pathways involved in energy homeostasis.

\section{References}

Aicher SA, Goldberg A, Sharma S, Pickel VM (2000) mu-opioid receptors are present in vagal afferents and their dendritic targets in the medial nucleus tractus solitarius. J Comp Neurol 422:181-190.

Blevins JE, Stanley BG, Reidelberger RD (2000) Brain regions where cholecystokinin suppresses feeding in rats. Brain Res 860:1-10.

Bodnar RJ, Hadjimarkou MM (2002) Endogenous opiates and behavior: 2001. Peptides 23:2307-2365.

Broberger C, Hokfelt T (2001) Hypothalamic and vagal neuropeptide circuitries regulating food intake. Physiol Behav 74:669-682.

Broberger C, Holmberg K, Shi TJ, Dockray G, Hokfelt T (2001) Expression and regulation of cholecystokinin and cholecystokinin receptors in rat nodose and dorsal root ganglia. Brain Res 903:128-140.

Bronstein DM, Schafer MK, Watson SJ, Akil H (1992) Evidence that betaendorphin is synthesized in cells in the nucleus tractus solitarius: detection of POMC mRNA. Brain Res 587:269-275.

Clement K, Vega N, Laville M, Pelloux V, Guy-Grand B, Basdevant A, Vidal H (2002) Adipose tissue gene expression in patients with a loss of function mutation in the leptin receptor. Int J Obes Relat Metab Disord 26:1533-1538.

Coll AP, Farooqi IS, Challis BG, Yeo GS, O’Rahilly S (2004) Proopiomelanocortin and energy balance: insights from human and murine genetics. J Clin Endocrinol Metab 89:2557-2562.

Cone RD, Cowley MA, Butler AA, Fan W, Marks DL, Low MJ (2001) The arcuate nucleus as a conduit for diverse signals relevant to energy homeostasis. Int J Obes Relat Metab Disord 25 [Suppl 5]:S63-S67.

Corp ES, McQuade J, Moran TH, Smith GP (1993) Characterization of type A and type B CCK receptor binding sites in rat vagus nerve. Brain Res 623:161-166.

Cowley MA, Smart JL, Rubinstein M, Cerdan MG, Diano S, Horvath TL, Cone RD, Low MJ (2001) Leptin activates anorexigenic POMC neurons through a neural network in the arcuate nucleus. Nature 411:480-484.

Doyle MW, Andresen MC (2001) Reliability of monosynaptic sensory transmission in brain stem neurons in vitro. J Neurophysiol 85:2213-2223.

Doyle MW, Bailey TW, Jin YH, Appleyard SM, Low MJ, Andresen MC (2004) Strategies for cellular identification in nucleus tractus solitarius slices. J Neurosci Methods 137:37-48.

Fan W, Ellacott KL, Halatchev IG, Takahashi K, Yu P, Cone RD (2004) Cholecystokinin-mediated suppression of feeding involves the brainstem melanocortin system. Nat Neurosci 7:335-336.

Faulconbridge LF, Cummings DE, Kaplan JM, Grill HJ (2003) Hyperphagic effects of brainstem ghrelin administration. Diabetes 52:2260-2265.

Grill HJ, Kaplan JM (2002) The neuroanatomical axis for control of energy balance. Front Neuroendocrinol 23:2-40.

Grill HJ, Schwartz MW, Kaplan JM, Foxhall JS, Breininger J, Baskin DG (2002) Evidence that the caudal brainstem is a target for the inhibitory effect of leptin on food intake. Endocrinology 143:239-246.

Grill HJ, Carmody JS, Amanda Sadacca L, Williams DL, Kaplan JM (2004) Attenuation of lipopolysaccharide anorexia by antagonism of caudal brainstem but not forebrain GLP-1-R. Am J Physiol Regul Integr Comp Physiol 287:R1190-R1193.

Gross PM, Wall KM, Pang JJ, Shaver SW, Wainman DS (1990) Microvascular specializations promoting rapid interstitial solute dispersion in nucleus tractus solitarius. Am J Physiol 259:R1131-R1138.
Ibrahim N, Bosch MA, Smart JL, Qiu J, Rubinstein M, Ronnekleiv OK, Low MJ, Kelly MJ (2003) Hypothalamic proopiomelanocortin neurons are glucose responsive and express K(ATP) channels. Endocrinology 144:1331-1340.

Joseph SA, Pilcher WH, Bennett-Clarke C (1983) Immunocytochemical localization of ACTH perikarya in nucleus tractus solitarius: evidence for a second opiocortin neuronal system. Neurosci Lett 38:221-225.

Kirkham TC, Blundell JE (1984) Dual action of naloxone on feeding revealed by behavioural analysis: separate effects on initiation and termination of eating. Appetite 5:45-52.

Kotz CM, Billington CJ, Levine AS (1997) Opioids in the nucleus of the solitary tract are involved in feeding in the rat. Am J Physiol 272:R1028-R1032.

Kotz CM, Glass MJ, Levine AS, Billington CJ (2000) Regional effect of naltrexone in the nucleus of the solitary tract in blockade of NPY-induced feeding. Am J Physiol Regul Integr Comp Physiol 278:R499-R503.

Lankisch TO, Tsunoda Y, Lu Y, Owyang C (2002) Characterization of CCK(A) receptor affinity states and $\mathrm{Ca}^{(2+)}$ signal transduction in vagal nodose ganglia. Am J Physiol Gastrointest Liver Physiol 282:G1002-G1008.

Levine AS, Billington CJ (2004) Opioids as agents of reward-related feeding: a consideration of the evidence. Physiol Behav 82:57-61.

Moran TH, Norgren R, Crosby RJ, McHugh PR (1990) Central and peripheral vagal transport of cholecystokinin binding sites occurs in afferent fibers. Brain Res 526:95-102.

Moran TH, Ladenheim EE, Schwartz GJ (2001) Within-meal gut feedback signaling. Int J Obes Relat Metab Disord 25 [Suppl 5]:S39-S41.

Moriarty P, Dimaline R, Thompson DG, Dockray GJ (1997) Characterization of cholecystokininA and cholecystokininB receptors expressed by vagal afferent neurons. Neuroscience 79:905-913.

Palkovits M, Eskay RL (1987) Distribution and possible origin of betaendorphin and ACTH in discrete brainstem nuclei of rats. Neuropeptides 9:123-137.

Palkovits M, Mezey E, Eskay RL (1987) Pro-opiomelanocortin-derived peptides (ACTH/beta-endorphin/alpha-MSH) in brainstem baroreceptor areas of the rat. Brain Res 436:323-338.

Paxinos F (2001) The mouse brain in stereotaxic coordinates, Ed 2. New York: Academic.

Reidelberger RD, Hernandez J, Fritzsch B, Hulce M (2004) Abdominal vagal mediation of the satiety effects of CCK in rats. Am J Physiol Regul Integr Comp Physiol 286:R1005-R1012.

Ritter RC (2004) Increased food intake and CCK receptor antagonists: beyond abdominal vagal afferents. Am J Physiol Regul Integr Comp Physiol 286:R991-R993.

Schwartz GJ (2000) The role of gastrointestinal vagal afferents in the control of food intake: current prospects. Nutrition 16:866-873.

Schwartz GJ, Moran TH (1996) Sub-diaphragmatic vagal afferent integration of meal-related gastrointestinal signals. Neurosci Biobehav Rev 20:47-56.

Schwartzberg DG, Nakane PK (1983) ACTH-related peptide containing neurons within the medulla oblongata of the rat. Brain Res 276:351-356.

Spiegelman BM, Flier JS (2001) Obesity and the regulation of energy balance. Cell 104:531-543.

Takagi H, Mizuta H, Matsuda T, Inagaki S, Tateishi K, Hamaoka T (1984) The occurrence of cholecystokinin-like immunoreactive neurons in the rat neostriatum: light and electron microscopic analysis. Brain Res 309:346-349.

Talman WT, Andreasen K, Calvin J, Eversmann-Johanns S (1991) Cholecystokinin in nucleus tractus solitarii modulates tonic and phasic gastric pressure. Am J Physiol 261:R217-R222.

Velley L, Milner TA, Chan J, Morrison SF, Pickel VM (1991) Relationship of Met-enkephalin-like immunoreactivity to vagal afferents and motor dendrites in the nucleus of the solitary tract: a light and electron microscopic dual labeling study. Brain Res 550:298-312.

Verberne AJ, Saita M, Sartor DM (2003) Chemical stimulation of vagal afferent neurons and sympathetic vasomotor tone. Brain Res Brain Res Rev 41:288-305.

Versteeg DH, Van Bergen P, Adan RA, De Wildt DJ (1998) Melanocortins and cardiovascular regulation. Eur J Pharmacol 360:1-14.

Zheng Z, Lewis MW, Travagli RA (2005) In vitro analysis of the effects of cholecystokinin (CCK) on rat brainstem motorneurons. Am J Physiol Gastrointest Liver Physiol, in press. 